\title{
The Helicobacter pylori VacA toxin is a urea permease that promotes urea diffusion across epithelia
}

\author{
Francesco Tombola, ${ }^{1}$ Laura Morbiato, ${ }^{1}$ Giuseppe Del Giudice, ${ }^{2}$ Rino Rappuoli, ${ }^{2}$ \\ Mario Zoratti, ${ }^{1}$ and Emanuele Papini ${ }^{3}$ \\ ${ }^{1}$ Centro Consiglio Nazionale delle Ricerche Biomembrane, Department of Biomedical Sciences, \\ University of Padova, Padova, Italy \\ ${ }^{2}$ Immunobiology Research Institute Siena, Siena, Italy \\ ${ }^{3}$ Department of Biomedical Sciences and Human Oncology, Section of General Pathology, \\ University of Bari, Bari, Italy \\ Address correspondence to: Emanuele Papini, Department of Biomedical Sciences and Human Oncology, \\ Section of General Pathology, University of Bari, Piazza Giulio Cesare 11, 70124 Bari, Italy. \\ Phone: 39-080-5478465; Fax: 39-080-5478471; E-mail: papinie@civ.bio.unipd.it. \\ Received for publication April 16, 2001, and accepted in revised form July 24, 2001.
}

\begin{abstract}
Urease and the cytotoxin VacA are two major virulence factors of the human pathogen Helicobacter pylori, which is responsible for severe gastroduodenal diseases. Diffusion of urea, the substrate of urease, into the stomach is critically required for the survival of infecting $H$. pylori. We now show that VacA increases the transepithelial flux of urea across model epithelia by inducing an unsaturable permeation pathway. This transcellular pathway is selective, as it conducts thiourea, but not glycerol and mannitol, demonstrating that it is not due to a loosening of intercellular junctions. Experiments performed with different cell lines, grown in a nonpolarized state, confirm that VacA permeabilizes the cell plasma membrane to urea. Inhibition studies indicate that transmembrane pores formed by VacA act as passive urea transporters. Thus, their inhibition by the anion channel blocker 5-nitro-2-(3phenylpropylamino) benzoic acid significantly decreases toxin-induced urea fluxes in both polarized and nonpolarized cells. Moreover, phloretin, a well-known inhibitor of eukaryotic urea transporters, blocks VacA-mediated urea and ion transport and the toxin's main biologic effects. These data show that VacA behaves as a low- $\mathrm{pH}$ activated, passive urea transporter potentially capable of permeabilizing the gastric epithelium to urea. This opens the novel possibility that in vivo VacA may favor $H$. pylori infectivity by optimizing urease activity.
\end{abstract}

J. Clin. Invest. 108:929-937 (2001). DOI: 10.1172/JCI200113045.

\section{Introduction}

Infection by Helicobacter pylori $(1,2)$ determines an increased incidence of gastritis, gastroduodenal ulcer, and gastric adenocarcinoma and lymphomas in humans. The colonization of the stomach by this microorganism requires complex adaptation processes. Among them, a key feature is acid tolerance allowing the bacterium to cross the stomach lumen ( $\mathrm{pH} 1-2)$ and to survive within the mucous layer covering the gastric epithelium ( $\mathrm{pH}$ range, 1-2 to 6). Cell death by acidification is prevented by a cytosolic urease, which converts urea into ammonia and bicarbonate (1-6). These products of urea hydrolysis are also metabolic substrates for $\mathrm{H}$. pylori $(7,8)$. The importance of urea availability for $H$. pylori is confirmed by the presence of an acid-activated passive urea transporter (UreI) in the bacterial inner membrane $(9,10)$ and by positive chemotaxis of the parasite toward urea $(11,12)$.

Besides urease, efficient gastric colonization by $H$. pylori also requires the cytotoxin $\operatorname{VacA}(13)$. This approximately $88-\mathrm{kDa}$ secreted protein is a major virulence and pathogenic factor, linked to severe gastric tissue damage
(14). In vitro, VacA needs activation by acidic $\mathrm{pH}$ pretreatment (15) and induces cell vacuolation and various cytotoxic effects $(2,14,16)$. VacA forms anion-selective channels in model and cell membranes (17-20). However, the in vivo relevance of these phenomena for $H$. pylori gastric infection and alteration is still undefined.

We investigated whether VacA cooperates with urease by increasing the diffusion of urea from the mucosal tissue to the stomach lumen. This hypothesis is partly based on previous studies suggesting that VacA's function in vivo is the selective permeabilization of the gastric epithelium to nutrients and other useful molecules, by altering cell-cell junctions $(21,22)$ and/or cell membranes $(17,20)$. By using polarized and nonpolarized cells and model membranes, and by combining functional, pharmacological, and electrophysiological approaches, it is shown that VacA determines a rapid and selective increase of epithelial permeability to urea, through the generation of a passive and unsaturable transcellular pathway. Evidence is provided that formation of VacA pores in the cell plasma membrane is responsible for this increased urea permeability. 


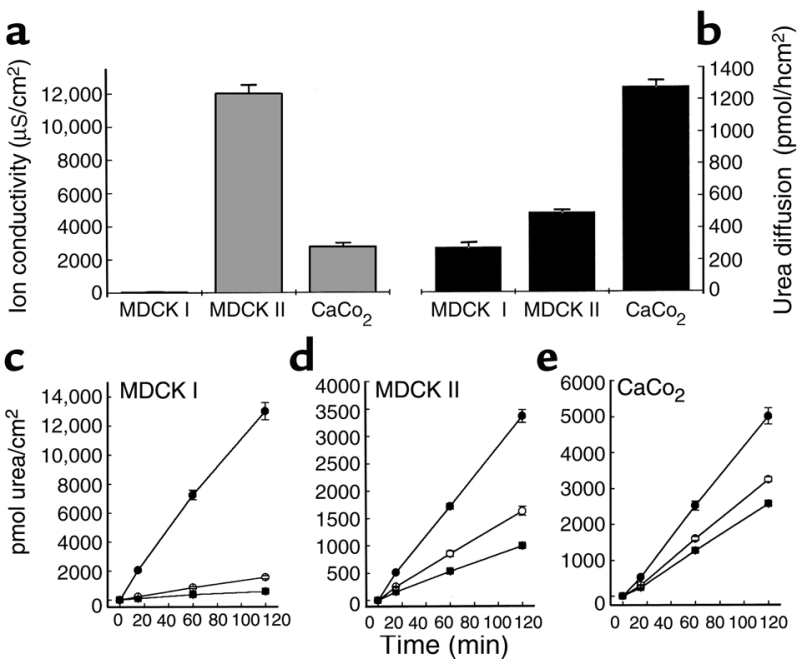

\section{Figure 1}

Effect of VacA on urea transepithelial fluxes in polarized MDCK I, MDCK II, and $\mathrm{CaCo}_{2}$ cell monolayers. (a) Cell monolayers were differentiated in vitro on polycarbonate porous filters, and their ion conductivity was determined at $37^{\circ} \mathrm{C}$. (b) After addition of $45 \mu \mathrm{M}$ $\left[{ }^{14} \mathrm{C}\right]$ urea to the basolateral compartment, urea transepithelial flux was measured at $37^{\circ} \mathrm{C}$, determining the amount of tracer in the apical compartment. (c-e) Cell monolayers were incubated apically with $125 \mathrm{nM}$ preactivated $(\mathrm{pH} 2.0)$ VacA (filled circles), nonactivated VacA (open circles) or with no toxin (filled squares) in DMEM, $10 \%$ FCS. Transepithelial diffusion of $\left[{ }^{14} \mathrm{C}\right]$ urea into the apical compartment was determined after different time intervals, beginning at 3 hours. Values are the mean of three experiments \pm SE.

\section{Methods}

Reagents. VacA from strain CCUG 17874 was purified as described previously (23) and was stored at $4^{\circ} \mathrm{C}$ in PBS ( $\mathrm{pH} 7.4$ ). VacA was activated by treatment at $\mathrm{pH}$ $2.0,37^{\circ} \mathrm{C}$ for 3 minutes. AGS and MKN28 cells were a kind gift from V. Ricci (Institute of Human Physiology, University of Pavia, Pavia, Italy). Plastic-ware, culture media, and FCS were from Life Technologies (Milan, Italy). Transwell polycarbonate filters for in vitro epithelial differentiation (pore diameter, $0.4 \mu \mathrm{m}$ ) were from Corning-Costar Corp. (Cambridge, Massachusetts, USA). $\left[{ }^{14} \mathrm{C}\right]$ Urea $(57 \mathrm{mCi} / \mathrm{mmol}), \mathrm{D}$ $\left[{ }^{14} \mathrm{C}\right]$ mannitol $(56 \mathrm{mCi} / \mathrm{mmol})$, and $\left[1(3)-{ }^{3} \mathrm{H}\right]$ glycerol (3 Ci/mmol) were from Amersham Pharmacia Biotech (Milan, Italy). $\left[{ }^{14} \mathrm{C}\right]$ Thiourea $(53 \mathrm{mCi} / \mathrm{mmol})$ was from NEN Life Science Products (Boston, Massachusetts, USA). The 5-nitro-2-(3-phenylpropylamino) benzoic acid (NPPB), phloretin, parachloromercury-benzensulfonate (pCMBS), ouabain, 8Br-cAMP, 8Br-cGMP, collagenase A1, thiourea, and urea were from SigmaAldrich srl (Milan, Italy). Synthetic diphytanoyl-phosphatidylcholine (purity >99\%) was from Avanti Polar Lipids (Alabaster, Alabama, USA). Urea solutions were made freshly before experiments.

Cell culture. Cells were grown on plastic at $37^{\circ} \mathrm{C}$ in a humidified 5\% (vol/vol) $\mathrm{CO}_{2}$ atmosphere in DMEM containing 10\% (vol/vol) FCS and gentamycin. TrypsinEDTA detached cells were seeded on polycarbonate fil- ters or plastic wells at the density of $0.5 \times 10^{6} / \mathrm{cm}^{2}$ and further kept in culture medium as indicated above. Epithelial differentiation was obtained after culturing on filters for 14 days, whereas nondifferentiated cells were cultured on plastic for 2 days before experiments.

Measurement of transepithelial fluxes. Monolayers on filters were washed with fresh culture medium and treated with acid-activated or nonactivated VacA (15-125 $\mathrm{nM})$ diluted in the same medium. Controls were treated in the same way with equivalent aliquots of PBS. After $0.25-27.0$ hours of incubation, unbound toxin was washed out by rinsing with DMEM without phenol red, 0.023\% BSA (wt/vol), 10 mM Na-HEPES (pH 7.4), and incubation was continued in the same medium at $37^{\circ} \mathrm{C}$, in normal atmosphere, after placing radiolabeled tracers $(45 \mu \mathrm{M}$ solutes or $2 \mu \mathrm{Ci} / \mathrm{ml}$ in the case of $\left[{ }^{3} \mathrm{H}\right]$ water) in the basolateral chamber. After the desired time intervals, the apical medium was retrieved and replaced by prewarmed medium. Radioactivity was determined by liquid scintillation and flows expressed as $\mathrm{pmol} / \mathrm{hcm}^{2}$ of cell monolayer. Ion conductivity was measured at $37^{\circ} \mathrm{C}$ with a Millipore Corp. apparatus (Bedford, Massachusetts, USA). In some experiments, unlabeled urea and/or thiourea (100-400 $\mathrm{mM})$, NPPB, pCMBS, or phloretin (50-500 $\mu \mathrm{M})$ was included in both apical and basolateral chambers during the transepithelial diffusion assays, or during VacA intoxication, as specified.

Measurement of transmembrane fluxes. Cells in plastic wells were intoxicated, or not, with VacA as described above. In some cases, cells were washed twice with icecold PBS ( $\mathrm{pH} 7.4$ ), containing $0.023 \%$ (wt/vol) BSA (PBS-BSA) and further incubated with radiolabeled solutes $(45 \mu \mathrm{M})$ in the same medium at $4{ }^{\circ} \mathrm{C}$ for different time intervals (2.5-15 minutes). Radioactivity associated to cells was determined after three washings (washing time, $\sim 20$ seconds) with PBS-BSA $\left(4^{\circ} \mathrm{C}\right)$ and cell lysis in PBS-BSA plus $0.5 \%$ (wt/vol) SDS. Alternatively, after intoxication, cells were washed with culture medium and further incubated at $37^{\circ} \mathrm{C}$ in the same conditions for 1 hour in the presence of labeled tracers $(45 \mu \mathrm{M})$. Cells were then rapidly washed and further incubated in PBS-BSA at $4^{\circ} \mathrm{C}$. The radioactivity present in the extracellular medium was determined at various time points (2.5-15 minutes). In some experiments, AGS and HeLa cells were depleted of ATP (24): cells were pretreated at $37^{\circ} \mathrm{C}$ with 1 $\mathrm{mg} / \mathrm{ml} \mathrm{NaN}_{3}$ for 30 minutes and for an additional 30 minutes with $1 \mathrm{mg} / \mathrm{ml} \mathrm{NaN}_{3}$ and $13 \mathrm{mM}$ 2-deoxy-Dglucose in glucose- and pyruvate-free DMEM plus $10 \%$ (vol/vol) dialyzed FCS, and further incubated with VacA in the same conditions, before determining transmembrane urea fluxes.

In other experiments, control or VacA-intoxicated HeLa cells were pretreated with $1 \mathrm{mM} 8 \mathrm{Br}$-cAMP or 1 $\mathrm{mM} 8 \mathrm{Br}-\mathrm{cGMP}$ or $0.250 \mathrm{mM}$ ouabain for 30 minutes, and urea fluxes were then measured in the presence of the same agonists. Alternatively, during urea flux measurement, $\mathrm{NaCl}$ in the extracellular medium was 

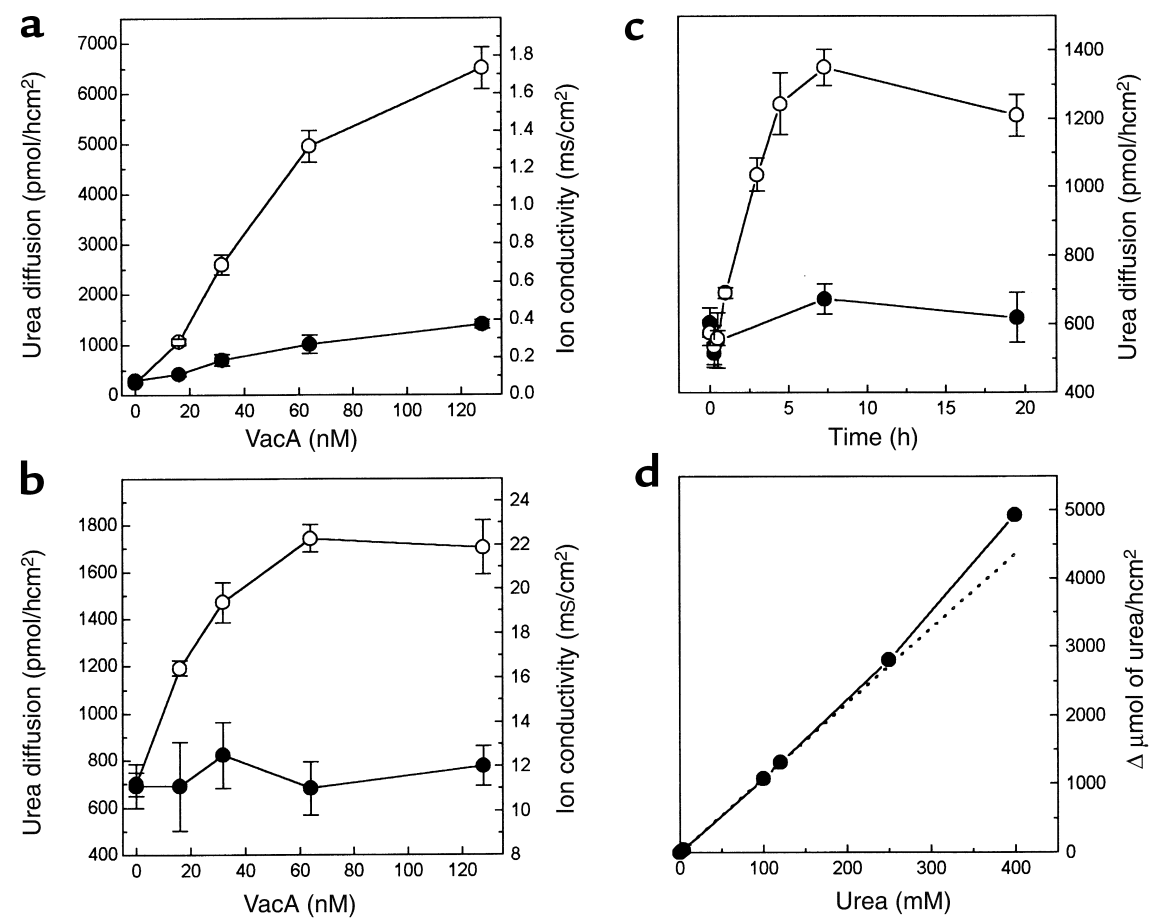

Figure 2

Dose response, kinetics, and unsaturability of VacA-induced urea transepithelial flux. Cell monolayers of MDCK I (a) and MDCK II (b) were apically treated with the indicated concentrations of activated VacA in DMEM, $10 \% \mathrm{FCS}$ for 3 hours at $37^{\circ} \mathrm{C}$. Transepithelial urea diffusion (open circles) and ion conductivity (filled circles) were determined. Values are the mean of four experiments in duplicate \pm SE. (c) After the indicated intoxication times with activated (open circles) or nonactivated (filled circles) VacA (125 nM), transepithelial urea flux across MDCK II cell polarized monolayers was determined. The averages of two experiments run in duplicate are reported with average deviations. (d) After a 3-hour treatment with VacA, as above, $45 \mu \mathrm{M}\left[{ }^{14} \mathrm{C}\right]$ urea plus the indicated concentration of cold urea was placed in the basolateral chamber. The amount of radioactivity in the apical chamber was then measured, yielding plots analogous to those in Figure $1, \mathrm{c}-\mathrm{e}$, whose slopes are plotted. The dotted line represents the theoretical linear correlation.

replaced by isoosmotic mannitol (25). Oocytes isolated from Xenopus laevis ovary were treated with 2 $\mathrm{mg} / \mathrm{ml}$ collagenase A1 in OR-2 buffer for 2 hours at $25^{\circ} \mathrm{C}$ and then defolliculated manually. After an overnight recovery at $20^{\circ} \mathrm{C}$, oocytes were treated or not with acid activated VacA at $24^{\circ} \mathrm{C}$ for 4 hours, and the uptake and release of ${ }^{14} \mathrm{C}$-urea were determined as described (25) in Barth's buffer $(88 \mathrm{mM} \mathrm{NaCl}, 1 \mathrm{mM}$ $\mathrm{KCl}, 0.82 \mathrm{mM} \mathrm{MgSO}_{4}, 0.33 \mathrm{mM} \mathrm{Ca}\left(\mathrm{NO}_{3}\right)_{2}, 0.41 \mathrm{mM}$ $\mathrm{CaCl}_{2}, 2.4 \mathrm{mM} \mathrm{NaHCO} 3,10 \mathrm{mM}$ HEPES/Na [pH 7.4], $10 \mathrm{U} / \mathrm{ml}$ penicillin, and $10 \mu \mathrm{g} / \mathrm{ml}$ streptomycin) at $24^{\circ} \mathrm{C}$. Phloretin $(200 \mu \mathrm{M})$ was included in the urea flux experiments in some cases.

Cell vacuolation. Cells were seeded in 24-wells trays at the density of $10^{3} / \mathrm{cm}^{2} 2$ days before experiments and then treated with $50 \mathrm{nM}$ VacA preactivated at $\mathrm{pH} 2.0$ in DMEM containing $5 \mathrm{mM} \mathrm{NH}_{4} \mathrm{Cl}$ and various concentrations of phloretin $(0-480 \mu \mathrm{M})$ at $37^{\circ} \mathrm{C}$. After 6 hours, cell vacuolation was quantified using the neutral red uptake assay as described previously (15).

Measurement of VacA-induced ion current in planar lipid bilayers. Experiments were performed as described previously (17), with $0.5 \mathrm{M} \mathrm{KCl}, 0.5 \mathrm{mM} \mathrm{CaCl}_{2}, 0.5 \mathrm{mM}$ $\mathrm{MgCl}_{2}$, and $10 \mathrm{mM} \mathrm{HEPES} / \mathrm{K}$ (pH 7.2) in both chambers. VacA (2-4 nM) activated at a low pH (2.0) was added to the cis compartment, and current was deter- mined with or without addition to either the cis or the trans side of inhibitors $(0-200 \mu \mathrm{M}$ phloretin; 0-500 $\mu \mathrm{M}$ pCMBS; 0-400 mM thio-urea and urea). The current was carried mainly by $\mathrm{Cl}^{-}$, as $\mathrm{VacA}$ is anion selective (17).

\section{Results}

VacA increases the transepithelial diffusion of urea. Polarized epithelia in vitro mimic the watertight epithelia present in the kidney, the bladder and, most relevant for the present study, the gastric mucosa (26). Tightness is associated with the development of a differen-

\section{Table 1}

Effect of VacA on the diffusion rate $\left(\mathrm{pmol} / \mathrm{hcm}^{2}\right)$ of various molecules across MDCK II monolayers

$\begin{array}{lccc} & \text { CTRL } & \text { VacA } & \text { VacA-CTRL } \\ \text { Urea } & 597 \pm 45 & 1681 \pm 98 & 1084 \pm 143 \\ \text { Thiourea } & 841 \pm 89 & 2848 \pm 103 & 2007 \pm 192 \\ \text { Glycerol } & 601 \pm 67 & 701 \pm 18 & 100 \pm 85 \\ \text { Mannitol } & 840 \pm 61 & 862 \pm 72 & 22 \pm 133\end{array}$

The medium bathing the basolateral side of MDCK II monolayers was supplemented with the indicated radioactive tracers $(45 \mu \mathrm{M})$, and radioactivity in the apical chamber was measured as a function of time. Transepithelial diffusion of control- and $125 \mathrm{nM}$ of VacA-treated monolayers and their difference are reported, expressed as pmol/ $\mathrm{hcm}^{2} \pm \mathrm{SE}(n=3$ or 4$)$. 

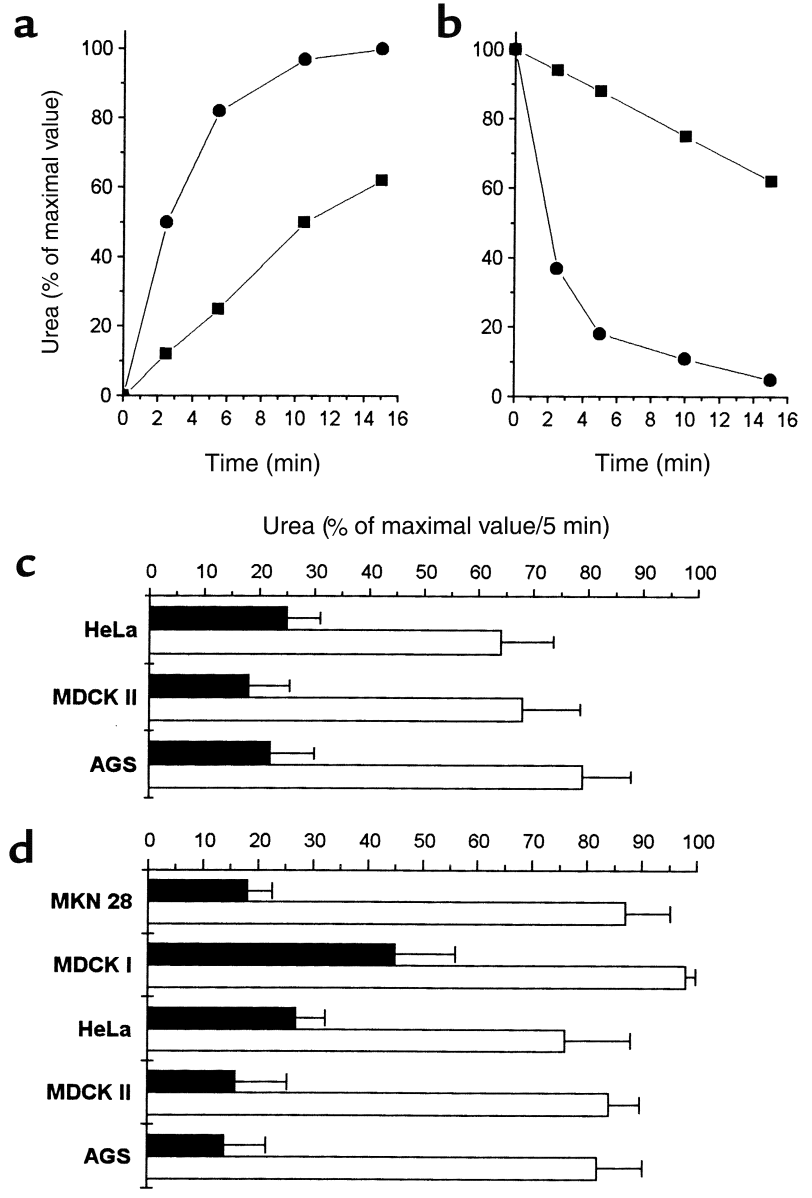

Figure 3

Effect of VacA on the plasma membrane permeability to urea in different nonpolarized cell types. ( $\mathbf{a}$ and $\mathbf{b}$ ) In the representative experiments shown $(n=10)$, AGS cells were grown on plastic for 2 days and treated (filled circles) or not (filled squares) for 3 hours with activated VacA $(125 \mathrm{nM})$ at $37^{\circ} \mathrm{C}$ in DMEM, 10\% FCS, washed with PBS-BSA at $4{ }^{\circ} \mathrm{C}$ and further incubated in the same medium. Influx of $\left[{ }^{14} \mathrm{C}\right]$ urea into cells was determined at the desired time intervals, after cell solubilization with $0.5 \%$ SDS (a). Alternatively, cells were equilibrated with $\left[{ }^{14} \mathrm{C}\right]$ urea in DMEM, $10 \% \mathrm{FCS}$ for 1 hour at $37^{\circ} \mathrm{C}$, and cell-associated radioactivity was determined as above after washing with PBS-BSA at $4{ }^{\circ} \mathrm{C}$ and further incubation (b). (c and d) The rate of cell urea influx or efflux (expressed as percent of maximal content after the first 5 minutes of assay) was determined in control (filled bars) or VacA-intoxicated (open bars) nondifferentiated cell lines, as specified. Values are the mean from at least three experiments run in duplicate \pm SE.

tiated apical membrane, enriched in cholesterol and sphingolipids $(27,28)$. Polarized MDCK I, MDCK II, and $\mathrm{CaCO}_{2}$ monolayers were used to test the effect of VacA on the transepithelial diffusion of urea and other small neutral molecules. Their basal resistance to urea diffusion was compared with transepithelial electrical resistance, a parameter largely dependent on the tightness of cell-cell junctions (the paracellular pathway). Data show that the epithelial permeabilities to urea and to ions do not correlate (Figure 1, a and b), strongly suggesting that the epithelial barrier to urea in our model systems is mostly represented by cell membranes, i.e., the transcellular pathway, in agreement with other studies $(26,28)$.

After intoxication for 3 hours with low $\mathrm{pH}$-activated VacA (125 nM), added to the apical compartment to mimic the in vivo conditions, urea diffusion increased in all tested epithelia (Figure 1, c, d, and e). The strongest effect ( 20-fold increment with respect to control epithelia) was recorded with MDCK I monolayers, whereas with MDCK II and $\mathrm{CaCo} 2$ cells, the effect was less pronounced (approximately two and three times the control value, respectively) but still significant. In absolute terms, the VacA effect with these two latter cell lines was $20-24 \%$ of that observed with MDCK I cells. Nonactivated VacA was much less effective in all cases.

Dose-response analysis of the effect of VacA on urea transepithelial flux and on the epithelial ion conductivity was performed with MDCK I and MDCK II epithelia after a 3-hour intoxication (Figure 2, a and b). In the former case, the VacA effect reached a maximal value at approximately $130 \mathrm{nM}$, and the half-maximal increase was obtained at approximately $40 \mathrm{nM}$. In the latter, a plateau was reached at approximately $60 \mathrm{nM}$, and the half-maximal concentration was approximately $20 \mathrm{nM}$. The epithelial ion conductivity of MDCK I monolayers increased by a factor of four with $125 \mathrm{nM} \mathrm{VacA}$, in agreement with previous observations $(21,22)$. However, at lower VacA doses $(<16 \mathrm{nM})$, the increase of ion conductivity was negligible, whereas urea flux was already four times higher than in control. In agreement with our previous data (21), VacA did not alter the TER of $\mathrm{CaCo} 2$ (data not shown) and MDCK II monolayers. These data suggest that VacA increases the transepithelial diffusion of urea by activating a transcellular pathway, although some contribution of the paracellular pathway probably occurs with MDCK I cells at high VacA doses (see below).

Urea diffusion through MDCK II epithelia increased immediately after VacA addition and reached a maximum (threefold increase) after approximately 7-8 hours. Urea flow remained high for the following approximately 15 hours (Figure 2c). Nonactivated VacA had a lower effect even after a long incubation time.

To test whether the urea transport system induced by VacA was saturable, the flow of $\left[{ }^{14} \mathrm{C}\right]$ urea was measured with MDCK II monolayers in the presence of increasing concentrations of unlabeled urea (Figure 2d). Urea diffusion increased almost linearly up to $400 \mathrm{mM}$, consistent with a passive unsaturable urea transport.

Table 1 compares the effect of VacA on the transepithelial flow of urea and other small neutral molecules across MDCK II monolayers. The diffusion of thiourea was efficiently increased by VacA, whereas that of glycerol and mannitol was scarcely affected. As expected (21) mannitol diffusion was increased in MDCK I monolayers, but with a much lower efficacy $(\sim 6 \%$ of urea flow) (data not shown). The transepithelial flow of ${ }^{3} \mathrm{H}-\mathrm{H}_{2} \mathrm{O}$ was not modified (data not shown).

VacA increases the plasma membrane permeability to urea. The data described above suggest a VacA-elicited 
increase of the plasma membrane permeability to urea. Indeed, VacA increased the rate of urea penetration into nonpolarized human gastric cells (AGS), as well as its efflux after previous equilibration (Figure 3 , $a$ and $b$ ). The permeability to urea of the plasma membrane of nonpolarized MDCK I, MDCK II, HeLa, and MKN28 cells was also increased by VacA treatment (Figure 3, c and d). A dose response performed with AGS cells, after 3 hours of intoxication (Figure 4a), showed that VacAinduced permeabilization of the plasma membrane reached a plateau at approximately $125 \mathrm{nM}$, with a half maximal effect at approximately $30 \mathrm{nM}$. Similar data were obtained with nonpolarized MDCK II (data not shown). As in polarized epithelia, VacA also increased the transmembrane diffusion of thiourea, but not of glycerol (Figure 4a, inset). The effect of VacA on nonpolarized AGS cells (Figure 4b) was already strong after 15 minutes and almost maximal after 1 hour. In the following 7 hours, urea transmembrane flux remained constant, and it was not reversed by toxin washout (chase). A similar time course of permeabilization was found when urea uptake by cells was monitored (data not shown). The effect of VacA on AGS (Figure 4b, inset) and HeLa cells (data not shown) was not inhibited by the block of its endocytosis, after ATP depletion (24).

Neither the basal nor the VacA-induced urea fluxes could be modified by the presence of ouabain $(0.25$ $\mathrm{mM})$ or of $8 \mathrm{Br}$-cAMP $(1 \mathrm{mM})$ and $8 \mathrm{Br}-\mathrm{cGMP}(1 \mathrm{mM})$ (data not shown). Replacement of $\mathrm{NaCl}(150 \mathrm{mM})$ in the urea assay medium with $300 \mathrm{mM}$ mannitol did not affect VacA-induced urea fluxes (data not shown).

VacA-induced increase of transepithelial and transmembrane urea diffusion is due to the VacA pore. Anion-channel and urea transporter blockers were used to investigate the mechanism of VacA-induced urea permeation. NPPB, a blocker of the VacA pore $(20,29)$, inhibited toxininduced transepithelial flux of urea in MDCK II cell monolayers to a maximal extent of about $55 \%$ of controls, with a half-maximal concentration of about 50 $\mu \mathrm{M}$ (Figure 5a). Similarly, NPPB partially inhibited the plasma membrane permeabilization to urea induced by VacA in nonpolarized AGS cells (Figure 5b). Phloretin, a blocker of eukaryotic urea transporters (30), efficiently inhibited the increase of both transepithelial and transmembrane urea diffusion induced by VacA (Figure 5, a and b). The transmembrane urea flux of isolated oocytes from Xenopus laevis, intrinsically devoid of specific urea transport (31), was also increased $(35 \pm 4 \% ; n=3)$ by $\operatorname{VacA}\left(700 \mathrm{nM}\right.$ for 4 hours at $\left.24^{\circ} \mathrm{C}\right)$, and this effect was phloretin sensitive (data not shown).

Phloretin inhibited cell vacuolation of nonpolarized cells treated with VacA in the presence of ammonium ions (Figure 6a). The $\mathrm{pH}$ of acidic compartments in untreated cells was not altered by phloretin, as judged from the basal uptake of neutral red (data not shown). This compound also prevented the VacA-induced increase of ion conductivity of MDCK I cell monolayers (Figure 6b). In this epithelial system, urea flux was also inhibited by phloretin, with characteristics depending on the intoxication protocol (Figure 6c). If phloretin was coincubated with VacA and then washed out, or if it was added after intoxication, during the assay for urea transepithelial flux, a partial inhibition was observed. On the other hand, if it was maintained during both the intoxication and assay steps, a strong inhibition was observed, roughly corresponding to the sum of the effects elicited by the other protocols. This is consistent with inhibition by phloretin of both the
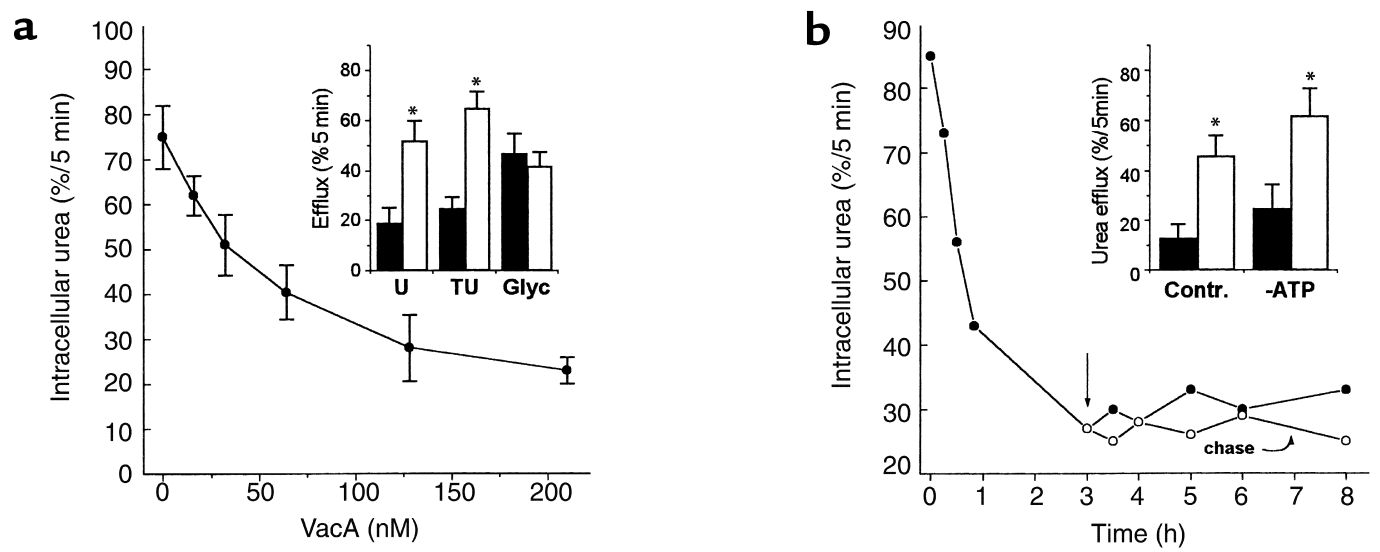

\section{Figure 4}

Dose response, selectivity, kinetics, and energy dependence of VacA-induced permeabilization of the plasma membrane of AGS cells. (a) Nonpolarized AGS cells were intoxicated with increasing concentration of VacA, and the rate of urea efflux was determined after equilibration. Inset: AGS cells were treated as above with VacA $(50 \mathrm{nM})$, washed, and incubated with PBS-BSA at $4^{\circ} \mathrm{C}$ supplemented with $45 \mu \mathrm{M}$ $\left[{ }^{14} \mathrm{C}\right]$ urea $(\mathrm{U}),\left[{ }^{14} \mathrm{C}\right]$ thiourea $(\mathrm{TU})$, or $\left[{ }^{3} \mathrm{H}\right]$ glycerol $(\mathrm{Glyc})$. The amount of released radioactivity was determined after 5 minutes. Data are the mean of three to four experiments run in duplicate \pm SE. (b) Nonpolarized AGS cells were incubated with $125 \mathrm{nM}$ of activated VacA for the indicated time intervals and then washed and assayed for $\left[{ }^{14} \mathrm{C}\right]$ urea release. The arrow indicates washing out of toxin before further incubation, in parallel samples (chase). Inset: AGS cells were or were not incubated under ATP-depleting conditions (see Methods) and treated for 3 hours with activated VacA (open bars) or left untreated (filled bars). The rate of urea efflux was then determined. The mean of three experiments $\pm \mathrm{SE}$ is reported. *The quantities compared are statistically different (Student's $t$ test; $P<0.05)$. Contr., control. 
$\mathbf{a}$

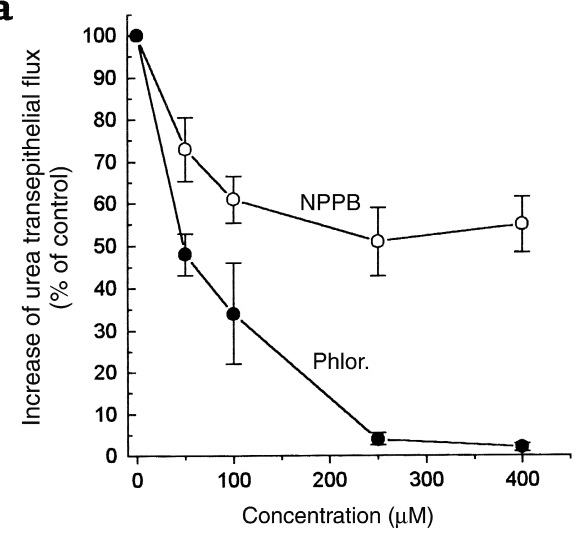

b

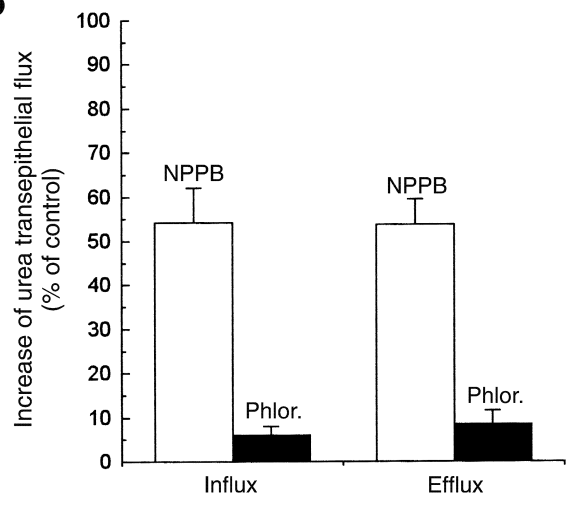

Figure 5

Effect of NPPB and phloretin (Phlor.) on the increase of transepithelial and transmembrane urea flux due to VacA. (a) Polarized MDCK II monolayers were treated with $125 \mathrm{nM}$ activated VacA for 3 hours and then washed and further assayed for $\left[{ }^{14} \mathrm{C}\right]$ urea transepithelial flow in the presence of the indicated concentrations of NPPB or phloretin (added to both apical and basolateral compartments). (b) Nonpolarized AGS cells were intoxicated by $125 \mathrm{nM}$ of VacA at $37^{\circ} \mathrm{C}$ for 3 hours, and assayed for initial rate of $\left[{ }^{14} \mathrm{C}\right]$ urea cell influx and efflux, in the presence of $250 \mu \mathrm{M}$ phloretin or NPPB. Data are expressed as percent of the increase observed in controls (+VacA, no inhibitor). Data are the mean of three experiments run in duplicate $\pm \mathrm{SE}$.

VacA-induced transcellular urea diffusion pathway and alteration of the paracellular pathway.

These data strongly suggest that phloretin directly interacts with the toxin. To test this possibility, its effect on the VacA anion channel was assessed in planar lipid bilayer experiments. Figure 7a shows that phloretin inhibited the VacA-induced ion current, with an $\mathrm{IC}_{50}$ of approximately $15 \mu \mathrm{M}$. Thiourea (Figure $7 \mathrm{~b}$ ) and pCMBS (data not shown), two other molecules known to affect passive urea transporters (32-34), were also tested. Thiourea $(400 \mathrm{mM})$ determined a $30 \%$ (trans) or a 45\% (cis) inhibition of ion conduction by VacA channels in planar lipid bilayers, depending on the side of addition. Urea $(400 \mathrm{mM})$ produced a weaker $5 \%$ inhibition. Consistently, $400 \mathrm{mM}$ thiourea inhibited VacAinduced $\left[{ }^{14} \mathrm{C}\right]$ urea diffusion across MDCK II monolayers by $70 \%$, whereas $400 \mathrm{mM}$ urea was ineffective. pCMBS (up to $250 \mu \mathrm{M}$ ) had no effect in either assay.

\section{Discussion}

Although recent data indicate that $H$. pylori relays in part upon the cytotoxin VacA for a successful coloniza- a

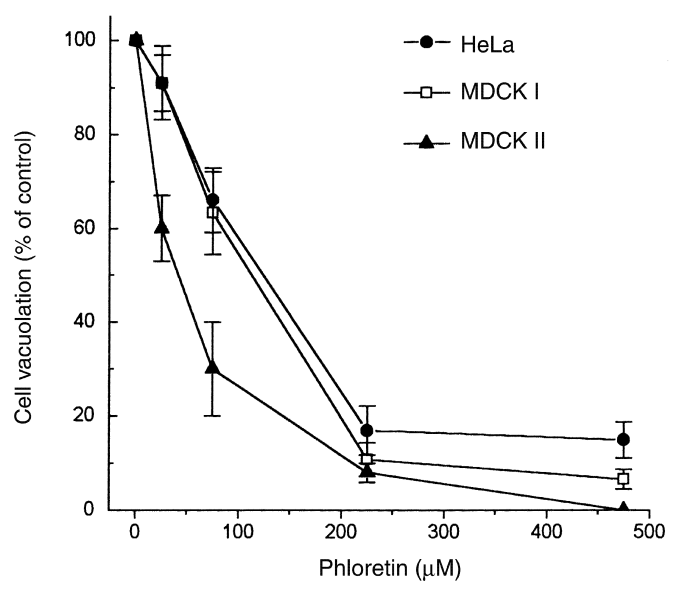

b

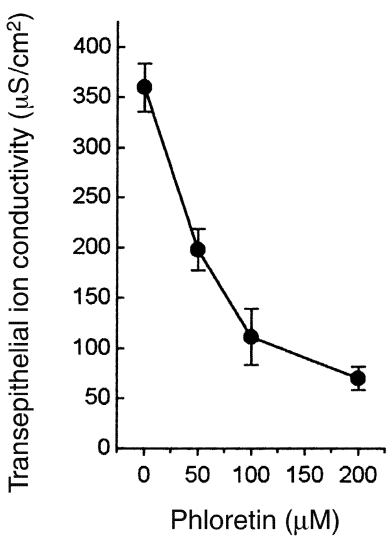

C

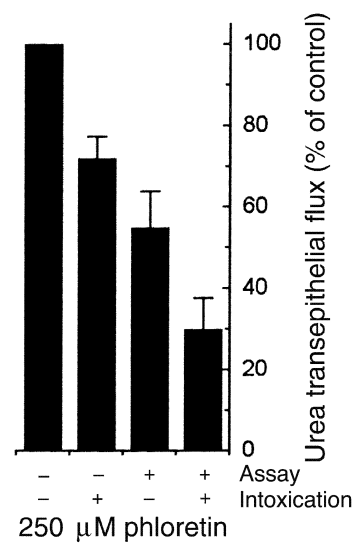

Figure 6

Effect of phloretin on cell vacuolation and increase of epithelial monolayer ion conductivity induced by VacA. Nonpolarized HeLa, MDCK I, and MDCK II cells were treated for 6 hours with $125 \mathrm{nM}$ of VacA in DMEM, 2\% FCS, plus $5 \mathrm{mM} \mathrm{NH}_{4} \mathrm{Cl}$ (a) or with medium alone. Cells were then assayed for uptake of neutral red. Data are expressed as percent of increase of NRU induced by VacA in the absence of inhibitors. Data are from three experiments run in duplicate \pm SE. (b) MDCK I monolayers were treated for 4 hours with $125 \mathrm{nM}$ VacA in DMEM $10 \%$ FCS at $37^{\circ} \mathrm{C}$ in the presence of different concentrations of phloretin. Ion conductivity was then measured. (c) The increase of basolateral to apical flow of $\left[{ }^{14} \mathrm{C}\right]$ urea was determined with polarized MDCK I epithelia treated with VacA for 4 hours, in the presence or absence of 250 $\mu \mathrm{M}$ phloretin during intoxication and/or urea flow assay, as specified. Values are expressed as percent of the effect induced by VacA in cell monolayers in the absence of phloretin and represent the mean of three experiments run in duplicate \pm SE. 
tion of the stomach (17), a clear link between the toxin biologic effects in vitro and its role in vivo is missing. The data presented here suggest that, in vivo, VacA action consists of enhancing the diffusion of urea from the under-mucosal tissue toward the stomach lumen. In fact, the epithelial barrier to urea diffusion is selectively decreased by VacA through the generation of a passive, unsaturable transport system. This action is not related to a decrease of the resistance of the paracellular pathway and is observed not only in high-resistance epithelia, such as MDCK I monolayers, but also in low-resistance ones, such as $\mathrm{CaCo} 2$ and MDCK II monolayers. These data indirectly suggest that, upon VacA intoxication, transcellular urea flow is increased. Experiments conducted with nonpolarized cells indeed indicate that VacA permeabilizes the cell plasma membrane to urea with negligible nonspecific membrane lesion, as the diffusion of other neutral molecules, such as is much less affected.

The rapid onset of the effect, not compatible with the endocytic rate of the toxin (24), and the lack of inhibition by block of endocytosis, indicate that VacA acts directly on the plasma membrane.

Although we cannot exclude that in polarized epithelial cells the basolateral membrane is also permeabilized by VacA, any such effect is likely to be irrelevant. This is because it is the apical membrane domain that poses the highest resistance to the flow of small nonelectrolytes $(26,35)$, owing to its external emi-leaflet particularly enriched in cholesterol, sphingomyelin, and glycosphingolipids $(26-28,35,36)$. In
MDCK cells, it has been estimated that the intrinsic resistance to urea of the apical emi-leaflet is about 50 times higher than that of the cytosolic emi-leaflet and much higher than that of the basolateral membrane (28). This implies that permeabilization of this lipid layer by VacA is necessary and sufficient to enhance urea flow through the entire epithelium.

In theory, VacA might permeabilize the plasma membrane to urea by activating an endogenous, urea transporter. Alternatively, given that low-pH activated VacA inserts into the lipid bilayer to form a transmembrane pore $(17,18,20)$, VacA itself may act as a passive urea transporter. In fact, the pharmacology of the VacAinduced effect, that is, the sensitivity to phloretin and the insensitivity to PCMBS, is compatible with both possibilities (30). The data presented in this work support this second view. In fact, inhibition of the VacA channel by NPPB $(20,29)$ substantially reduces also the urea transepithelial and transmembrane permeability increases determined by the toxin. Phloretin, a blocker of urea transporters, strongly reduces both urea diffusion and ion conduction due to the toxin, with comparable dose responses. As indicated by the lack of inhibitory effect of ouabain and of the absence of extracellular ions on VacA-dependent urea fluxes, there is no coupling between ion and urea transport, thus ruling out the indirect activation of an ion-coupled urea transporter by VacA. A further indication that VacAinduced urea flow is not coupled to $\mathrm{Na}^{+}$comes from the similarity of influx and efflux kinetics in cellular systems (Figure 3), despite the presence of a $\mathrm{Na}^{+}$electrochemical gradient.
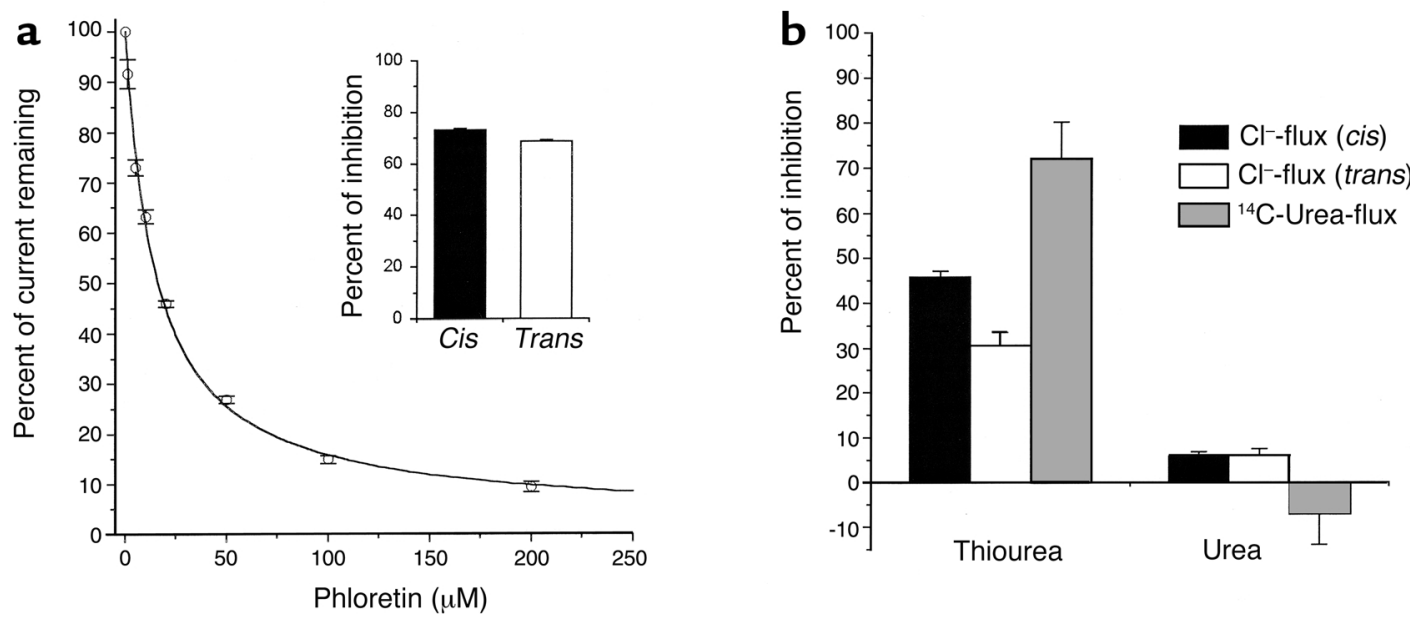

\section{Figure 7}

Effect of phloretin, thiourea, and urea on transmembrane current and transepithelial $\left[{ }^{14} \mathrm{C}\right]$ urea flux induced by VacA. (a) Activated ( $\left.\mathrm{pH} 2.0\right)$ VacA (2-4 nM) was added to planar diphytanoyl-phosphatidylcholine membranes, and the transmembrane current was measured before and after addition of increasing concentrations of phloretin to the cis side. The remaining current is reported as percent of the initial value. $\mathrm{V}_{\text {cis }}:-40 \mathrm{mV}$. Each point represents the mean of three to six experiments $\pm \mathrm{SE}$. A modified Langmuir's isotherm (38) was used for fitting; $K_{\mathrm{D}}=15 \pm 1 \mu \mathrm{M}$. Inset: The effect of $50 \mu \mathrm{M}$ phloretin on VacA-induced transmembrane current when added to the cis (filled column; $n=5$ ) or the trans (open column; $n=6$ ) side. (b) Inhibition of VacA-conducted current by $400 \mathrm{mM}$ thiourea or $400 \mathrm{mM}$ urea added to the cis (filled column; $n=4$ ) or to the trans side (open column; $n=4$ ) of the membrane. Gray columns represent the inhibitory effect of thiourea and urea on the increase of transepithelial diffusion of $\left[{ }^{14} \mathrm{C}\right]$ urea across polarized MDCK II cell monolayers, determined after a 3-hour intoxication by $125 \mathrm{nM}$ VacA. Data are expressed as the percent of inhibition with respect to the control in the absence of cold thiourea and urea and are the mean of three experiments run in duplicate \pm SE. 
As in the case of Xenopus oocytes, in which urea transporters are often expressed and characterized, no evidence of cAMP-activatable urea transporters was found in HeLa (see Results) or in MDCK cells (28). The VacAinduced urea flux was not modulated by cAMP either. Experiments on oocytes, believed to lack specific urea transporters, show that VacA induces a phloretin-sensitive increase of urea transmembrane flux also in this system, strengthening the notion that it acts as an autonomous transporter. In addition, the indirect activation of an aquaporin able to conduct also urea seems unlikely. The only mammalian aquaporin known not to be inhibited by thiol reagents is AQP-4, which does not conduct urea (37). In our model epithelial system, the transepithelial flow of water was not detectably increased by VacA intoxication.

In agreement with current ideas on VacA action (1), phloretin also interferes with VacA-induced cell vacuolation and TER decrease in MDCK I monolayers. Thiourea is conducted by the VacA-induced transport system and, at high concentrations, interferes with urea flow, suggesting that the two molecules enter the same pathway. Similarly, thiourea and, to minor extent, urea reduce ion conduction by VacA, indicating that these molecules engage the lumen of the VacA anion channel, interfering with ion flow.

A question that needs to be addressed is whether urea is limiting for $H$. pylori in the gastric environment. Although the stomach contains 1 - to $3-\mathrm{mM}$ urea, time- and space-dependent fluctuations of urea concentration may occur, as suggested by positive chemotaxis toward urea by $H$. pylori $(11,12)$. Moreover, urease activity itself is expected to determine a local urea shortage, especially in case of acid stress $(\mathrm{pH}<6.5)(9)$. Although an accurate analysis of the system is impossible at present, the surface density of $H$. pylori cells in mouse models $\left(10^{5}-10^{6} \mathrm{CFU} / \mathrm{stom}-\right.$ ach; ref. 13) and the measured rate of urea consumption by maximally activated urease (0.38-0.6 $\mu \mathrm{mol} / \mathrm{min} 10^{8} \mathrm{CFU}$; ref. 6) are compatible with a decrease of urea concentration within the thin mucous layer $(<50 \mu \mathrm{m})$ where bacterial cells reside. Under conditions of local urea depletion, the diffusion rate through the gastric barrier would be expected to be limiting, as our data show that basal urea equilibration through polarized cells takes hours. Hence, it is possible that the establishment of a high density of infecting cells is favored by an increased urea diffusion through the gastric mucosa.

These observations and considerations support the novel hypothesis that VacA, by increasing urea diffusion into the stomach, is part of an adaptation system, also comprising the urea transporter UreI and urease, aimed at enabling $H$. pylori to survive in the stomach. The evolution of pore-forming toxins as permeases selective for crucial substrates, capable of inserting in the membrane of host cells with no acute cytotoxicity, may be a relevant strategy also of other human pathogens associated to mucosal epithelia.
Gastric mucosa permeabilization may have other implications. Besides urea, the VacA transport system also conducts electrolytes and possibly other neutral solutes. As suggested by the loss of gastric integrity induced by barrier-breaking agents (ethanol, aspirin, detergents, phospholipases), this might predispose to tissue damage, given that the gastric epithelial barrier preserves the entire mucosa from the potentially deleterious effects of ingested food of extremely variable chemical composition and osmolarity (35). In conclusion, apical membrane permeabilization by VacA may favor $H$. pylori-associated diseases both by promotion of bacterial infection and by impairment of the capability of gastric cells to regulate their cytosolic milieu upon environmental variations.

\section{Acknowledgments}

The authors are grateful to Stefano Piccolo and his laboratory (University of Padua) for help with performing experiments with Xenopus oocytes. This research was supported by Cofin MURST 2000, by the University of Bari, and by CNR target project Biotechnology (97.01168.PF 49).

1. Montecucco, C., and Rappuoli, R. 2001. Living dangerously: how Helicobacter pylori survives in the human stomach. Nat. Rev. Mol. Cell Biol. 2:457-466.

2. Achtman, M., and Suerbaum, S. 2001. Helicobacter pylori: molecular and cellular biology. Horizon Scientific Press. Wymondham, United Kingdom.

3. Marshall, B.J., Barrett, L.J., Prakash, C., McCallum, R.W., and Guerrant, R.L. 1990. Urea protects Helicobacter (Campylobacter) pylori from the bactericidal effect of acid. Gastroenterology. 99:697-702.

4. Bauerfeind, P., Garner, R., Dunn, B.E., and Mobley, L.T. 1997. Synthesis and activity of Helicobacter pylori urease and catalase at low $\mathrm{pH}$. Gut. 40:25-30.

5. Scott, D.R., et al. 1998. The role of internal urease in acid resistance of Helicobacter pylori. Gastroenterology. 114:58-67.

6. Stingl, K., et al. 2000. Prolonged survival and cytoplasmic $\mathrm{pH}$ homeostasis of Helicobacter pylori at $\mathrm{pH}$ 1. Infect. Immun. 69:1178-1181.

7. Garner, R.M., Fulkerson, J., Jr, and Mobley, H.L. 1998. Helicobacter pylori glutamine synthetase lacks features associated with transcriptional and posttranslational regulation. Infect. Immun. 66:1839-1847.

8. Burns, B.P., Hazell, S.L., and Mendz, G.L. 1995. Acetyl-CoA carboxylase activity in Helicobacter pylori and the requirement of increased $\mathrm{CO}_{2}$ for growth. Microbiology. 141:3113-3118.

9. Gordon, D. 2000. A pH-sensitive channel regulates urea access to Helicobacter pylori urease. Gastroenterology. 118:249-250.

10. Weeks, D.L., Eskandari, S., Scott, R.D., and Sachs, G. 2000. A H ${ }^{+}$-gated urea channel: the link between Helicobacter pylori urease and gastric colonization. Science. 287:482-485.

11. Nakamura, H., et al. 1998. Urease plays an important role in the chemotactic motility of Helicobacter pylori in a viscous environment. Infect. Immun. 66:4832-4837.

12. Mizote, T., Yoshiyama, H., and Nakazawa, T. 1997. Urease-independent chemotactic responses of Helicobacter pylori to urea, urease inhibitors, and sodium bicarbonate. Infect. Immun. 65:1519-1521.

13. Salama, N.R., Otto, G., Tompkins, L., and Falkow, S. 2001. Vacuolating cytotoxin of Helicobacter pylori plays a role during colonization in a mouse model of infection. Infect. Immun. 69:730-736.

14. Montecucco, C., Papini, E., de Bernard, M., Telford, J.L., and Rappuoli, R. 1999. Helicobacter pylori vacuolating cytotoxin and associated pathogenic factors. In The comprehensive sourcebook of bacterial protein toxins. J.E. Alouf and J.H. Freer, editors. Academic Press Inc. San Diego, California, USA. 264-283.

15. de Bernard, M., et al. 1995. Low pH activates the vacuolating toxin of Helicobacter pylori, which becomes acid and pepsin resistant. J. Biol. Chem. 270:23937-23940.

16. Reyrat, J.-M., et al. 1999. Towards deciphering the Helicobacter pylori cytotoxin. Mol. Microbiol. 34:197-204.

17. Tombola, F., et al. 1999. Helicobacter pylori vacuolating toxin forms anionselective channels in planar lipid bilayers: possible implications for the mechanism of cellular vacuolation. Biophys. J. 76:1401-1409.

18. Iwamoto, H., Czajkowsky, D.M., Cover, T.L., Szabo, G., and Shao, Z. 
1999. VacA from Helicobacter pylori: a hexameric chloride channel. FEBS Lett. 450:101-104

19. Czajkowsky, D.M., Iwamoto, H., Cover, T.L., and Shao, Z. 1999. The vacuolating toxin from Helicobacter pylori forms hexameric pores in lipid bilayers at low pH. Proc. Natl. Acad. Sci. USA. 96:2001-2006.

20. Szabò, I., et al. 1999. Formation of anion-selective channels in the cell plasma membrane by the toxin VacA of Helicobacter pylori is required for its biological activity. EMBO J. 18:5517-5527.

21. Papini, E., et al. 1998. Selective increase of the permeability of polarized epithelial cell monolayers by Helicobacter pylori vacuolating toxin. J. Clin. Invest. 102:813-820.

22. Pelicic, V., et al. 1999. Helicobacter pylori VacA cytotoxin associated with the bacteria increases epithelial permeability independently of its vacuolating activity. Microbiology. 145:2043-2050.

23. Manetti, R, et al. 1995. Helicobacter pylori cytotoxin: importance of native conformation for induction of neutralizing antibodies. Infect. Immun. 63:4476-4480

24. McClain, M.S., Schraw, W., Ricci, V., Boquet, P., and Cover, T.L. 2000. Acid activation of Helicobacter pylori vacuolating cytotoxin (VacA) results in toxin internalization by eukaryotic cells. Mol. Microbiol. 37:433-442.

25. You, G., et al. 1993. Cloning and characterisation of the vasopressin-regulated urea transporter. Nature. 365:844-847.

26. Zeidel, M.L. 1996. Low permeabilities of apical membranes of barrier epithelia: what makes watertight membranes watertight? Am. J. Physiol. 271:F243-F245.

27. Lavelle, J.P., et al. 1997. Low permeabilities of MDCK cell monolayers: a model barrier epithelium. Am. J. Physiol. 273:F67-F75.

28. Hill, W.G., and Zeidel, M.L. 2000. Reconstituting the barrier properties of water-tight epithelial membrane by design of leaflet-specific liposomes. J. Biol. Chem. 275:30176-30185.

29. Tombola, F., et al. 1999. Inhibition of the vacuolating and anion channel activities of the VacA toxin of Helicobacter pylori. FEBS Lett. 460:221-225.

30. Sands, J.M., Timmer, R.T., and Gunn, R.B. 1997. Urea transporters in kidney and erythrocytes. Am. J. Physiol. 273:F321-F339.

31. Zhang, R., and Verkman, A.S. 1991. Water and urea permeability properties of Xenopus oocytes: expression of mRNA from toad urinary bladder. Am. J. Physiol. 260:C26-C34.

32. Couriaud, C., Ripoche, P., and Rousselet, G. 1996. Cloning and functional characterisation of a rat urea transporter: expression in the brain. Biochim. Biophys. Acta. 1309:197-199.

33. Martial, S., et al. 1996. Functional differentiation of human red blood cell and kidney urea transporters. Am J. Physiol. 271:F1264-F1268.

34. Couriaud, C., et al. 1999. Molecular and functional characterization of an amphibian urea transporter. Biochim. Biophys. Acta. 1421:347-352.

35. Priver, N.A., Rabon, E., and Zeidel, M.L. 1993. Apical membrane of the gastric parietal cell: water, proton, and nonelectrolyte permeabilities. Biochemistry. 32:2459-2468.

36. Van Meer, G., and Simons, K. 1986. The function of tight junctions in maintaining differences in lipid composition between the apical and the basolateral cell surface domains of MDCK cells. EMBO J. 5:1455-1464.

37. Borgnia, M., Nielsen, S., Engel, A., and Agre, P. 1999. Cellular and molecular biology of the aquaporin water channels. Annu. Rev. Biochem. 68:425-458.

38. Tombola, F., Del Giudice, G., Papini, E., and Zoratti, M. 2000. Blockers of VacA provide insights into the structure of the pore. Biophys. J. 79:863-873. 